Check for updates

Cite this: RSC Adv., 2017, 7, 53537

\title{
Effects of nano-grooved gelatin films on neural induction of human adipose-derived stem cells
}

\begin{abstract}
Chen-Yu Tsai, ${ }^{a}$ Chih-Ling Lin, ${ }^{a}$ Nai-Chen Cheng ${ }^{\star b}$ and Jiashing Yu (D)*a
The extra cellular matrix (ECM) and cell-cell interactions facilitate the survival, self-renewing and differentiation capabilities of stem cells. Biomaterials with specific structures such as grooves, ridges, pits, or pillars can mimic the topographic landscape of the niche. Cells can "sense" the mechanical properties and surface patterns, ranging from the micro- to nano-scale, of the substrate; hence, different sizes of nano-grooves on a gelatin surface were designed. The design of grooves can be systematically modified and those structures can reflect the organization of the ECM. In previous studies, polystyrene (PS) was often used because it is easy to fabricate topographic structures. For better biocompatibility, gelatin was chosen and fabricated into ideal nano-groove films. On the other hand, gelatin can be crosslinked by using several crosslinking agents, which leads to a higher mechanical strength and better flexibility. It was known that stem cells can serve as a source of neurons in transplantation therapies. The differentiation of neurons is associated with directionality of stem cell. To investigate the effect of topographic cues on stem cells, groove pattern arrays were constructed onto gelatin surfaces. Human adipose-derived stem cells (hASCs) were seeded onto the patterned gelatin films to observe cell proliferation and differentiation.
\end{abstract}

Received 15th August 2017

Accepted 6th November 2017

DOI: 10.1039/c7ra09020j

rsc.li/rsc-advances motility, and even the cell phenotype. ${ }^{6}$ The stiffness of tissues can be measured by the elastic modulus $E$ of a solid, which ranges from $0.5 \mathrm{kPa}$ (fat tissue) to $20000 \mathrm{kPa}$ (bone tissue). Comparing these values with a tissue culture on plastic or glass, where the elastic modulus is in the GPa range, it clearly shows that cells are not usually cultured in a non-physiological environment. As a result, many researchers have studied cells in vitro under a more physiological environment. For example, McDaniel et al. revealed that the stiffness of collagen fibrils can influence the phenotype of muscle cells. ${ }^{7}$ Synthetic substrates with controllable stiffness showed the differences in cell motility and adhesion. ${ }^{8}$ Besides, stiffness can further regulate cell lineage commitment and differentiation state, such as the differentiation of precursor cells into osteoblasts, neurites outgrowth and the striation of muscle cells. ${ }^{9}$

On the other hand, the effect of topography on cell behavior has been investigated since $1911 .{ }^{10}$ Cells can respond to topographic cues as small as $5 \mathrm{~nm}$. Therefore, topographic features from the micro- to the nano-scale have been widely developed. In general, surface topography is affected by roughness and patterns on the surface. ${ }^{11}$ Many types of features, for instance, grooves, dots and pillars, are developed, because these structures can be systematically modified and reflect the fibrillar organization of the ECM.

Among these structures, ridges and grooves have been investigated extensively. Micro- or nano-grooves can guide cells to align along the patterns. However, a previous study showed that fibroblasts do not align with groove depths below $35 \mathrm{~nm}$ or 
ridge widths smaller than $100 \mathrm{~nm} .{ }^{12}$ Yang et al. investigated the influence of nano-grooves on the morphology of osteoblast-like cells with a groove-to-ridge ratio of $1: 1$ (90-500 $\mathrm{nm}$ in width and $300 \mathrm{~nm}$ in depth). It showed an increased cell-spreading area compared to flat surfaces and elongated nuclei. ${ }^{\mathbf{1 3}}$ Sciancalepore et al. fabricated fibronectin (FN) micro-patterns to drive the differentiation of adult renal progenitor cells (ARPCs) in the absence of an exogenous chemical or cellular reprogramming. ${ }^{14}$

As mentioned above, there are lots of stem cells that can differentiate into neural cells including embryonic stem cells (ESCs), ${ }^{15-17}$ neural stem cells (NSCs) ${ }^{18-20}$ and adipose-derived stem cells (ASCs). ${ }^{21-24}$ Among these stem cells, ASCs are commonly used because they can be harvested easily and are able to differentiate into multiple lineages including adipocytes, ${ }^{25}$ myocytes, ${ }^{26}$ osteoblasts, ${ }^{27}$ chondrocytes ${ }^{28}$ and neural cells. ${ }^{29}$ Many studies have investigated how topography affect differentiation of human adipose-derived stem cells (hASCs). Deiwick et al. fabricated a "Lotus" structure using titanium to investigate how it affected the osteogenic differentiation of hASCs. ${ }^{30}$ In addition, Mobasseri et al. used polymers to fabricate grooves and studied neural differentiation of hASCs. ${ }^{31}$ However, materials used to fabricate topography are too rigid for cells.

In this study, to mimic the physiological environment of normal tissue, nano-grooves were fabricated onto a gelatin substrate, which is a natural and soft biomaterial. The mechanical properties of gelatin can be adjusted through crosslinking. Grooves were chosen because they can be fabricated into different sizes easily. To study the relationship between neural differentiation of stem cells and surface topography, hASCs were cultured on different groove sizes on gelatin films.

\section{Experimental section}

\section{Materials and methods}

Fabrication of nano-grooved gelatin films. Four sizes of grooved silicon substrates with a groove-to-ridge ratio of $1: 1$ (groove width/depth (nm): 400/100, 400/400, 800/100 and 800/ 400) were used to fabricate topography onto polydimethylsiloxane (PDMS, Dow Corning, USA) molds and gelatin films (Fig. 1). At first, the silicone rubber molds were fabricated by mixing the elastomer with a curing agent with a ratio of
$10: 1$. The mixture was cast onto silicon substrates and degassed to remove entrapped bubbles. After polymerizing at $80^{\circ} \mathrm{C}$ for $2 \mathrm{~h}$, PDMS molds with negative replicas of topography on substrates could be easily removed from the silicon substrates.

To fabricate the nano-grooved gelatin films, PDMS molds were placed and fixed in a Petri dish with the patterned side upwards. An aqueous solution of $5 \mathrm{wt} \%$ gelatin (Sigma-Aldrich, Germany) and 1\% PSA (Sigma-Aldrich, Germany) was cast into the Petri dish, and air-dried overnight at $25{ }^{\circ} \mathrm{C}$. The gelatin samples were subsequently immersed in binary solvent mixtures containing $N$-(3-dimethylaminopropyl)- $N^{\prime}$-ethylcarbodiimide hydrochloride (EDC, Sigma-Aldrich, Germany) and $N$-hydroxysuccinimide (NHS, Sigma-Aldrich, Germany). The reaction was allowed to proceed at $25{ }^{\circ} \mathrm{C}$ for $96 \mathrm{~h} .{ }^{32}$ Crosslinked gelatin films were cut down and thoroughly rinsed with deionized (DI) water to remove excess EDC and the urea byproduct. Before cell culturing, gelatin films were immersed in cell culture medium for $24 \mathrm{~h}$ to ensure the removal of excess crosslinking agent.

Topography measurement. To verify the fidelity of replication, the topography of silicon substrates was examined using a scanning electron microscope (SEM, NovaTM NanoSEM 23, FEI, USA). On the other hand, PDMS molds and nano-grooved gelatin films were examined using an atomic force microscope (AFM, Multimode 8, Bruker, USA). Gelatin films were immersed in DI water until fully swollen before measurement.

Crosslinking extent measurement. A previously reported assay was used to determine the number of uncrosslinked $\varepsilon^{-}$ amino groups in the crosslinked gelatin. ${ }^{33}$ Initially, $11 \mathrm{mg}$ of gelatin was mixed with $1 \mathrm{~mL}$ of $4 \% \mathrm{NaHCO}_{3}$ (Sigma-Aldrich, USA) and $1 \mathrm{~mL}$ of $0.5 \%$ 2,4,6-trinitrobenzenesulfonic acid (TNBS, Sigma-Aldrich, USA), and heated at $40^{\circ} \mathrm{C}$ for $4 \mathrm{~h} .3 \mathrm{~mL}$ of $6 \mathrm{~N} \mathrm{HCl}$ (Honeywell, USA) was added and the mixture was autoclaved for $1 \mathrm{~h}$ at $120^{\circ} \mathrm{C}$ and 15-17 psi. The hydrolysate was diluted with $5 \mathrm{~mL}$ of water, and then extracted with ethyl ether.

A $5 \mathrm{~mL}$ aliquot of the aqueous phase was removed from each sample and heated for $15 \mathrm{~min}$ in a hot water bath. After cooling to room temperature, samples were diluted again with $15 \mathrm{~mL}$ of water. The absorbance was measured at $346 \mathrm{~nm}$ in a UV/Vis spectrophotometer (Cary 100, Agilent, USA) against a blank. Four replicates were used in each determination. Blanks were prepared in triplicate by the same procedure as above, except
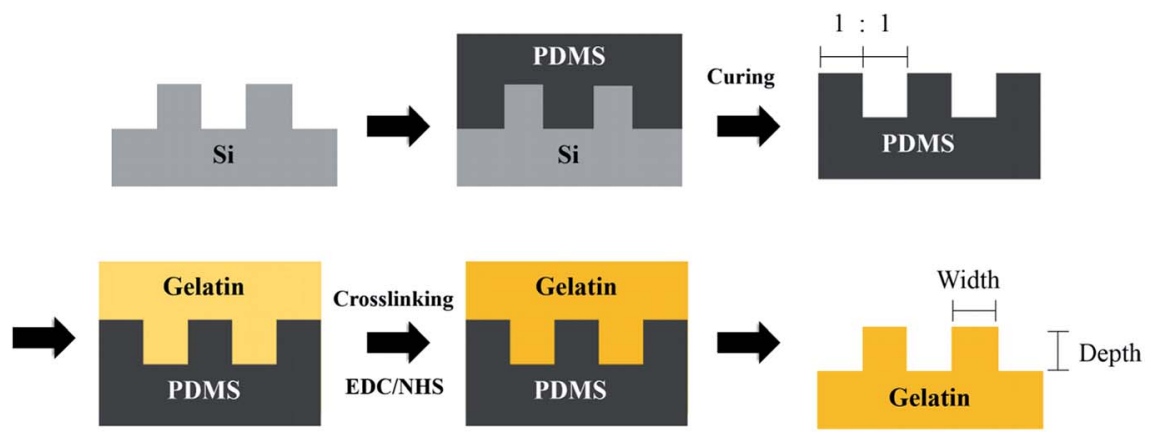

Fig. 1 Schematic illustration of the fabrication of nano-grooved gelatin films from silicon substrates. 
that $\mathrm{HCl}$ was added before TNBS to prohibit the reaction of amino groups with TNBS.

Mechanical test. Before the mechanical test, gelatin films with different crosslinking times were immersed in DI water until use. Gelatin films were cut into a round shape with an $8.1 \mathrm{~mm}$ diameter. The thickness of films was measured using a thickness gauge. Round gelatin films were compressed to half of their thickness by a mechanical test instrument (ElectroForce 3200 , TA, USA). The Young's modulus was calculated from the linear region of the stress-stain curve (initial slope: 0-10\%).

Swelling test. Gelatin films were weighed after being fully airdried. They were then immersed in DI water for different periods of time. Wet samples were wiped with filter paper to remove excess liquid and reweighed. The amount of adsorbed water was calculated as $W(\%)=100\left(\left(W_{\mathrm{w}}-W_{\mathrm{d}}\right) / W_{\mathrm{d}}\right)$, where $W_{\mathrm{w}}$ and $W_{\mathrm{d}}$ are the weights of the wet and dry samples.

Contact angle measurement. Nano-grooved and flat gelatin films were immersed in DI water before measurement. At first, gelatin film was fixed on the glass slide, and immersed in water with the patterned side down. Then, one air bubble was blown onto the patterned surface of the gelatin film using a microsyringe. After snapping clear images of the film and air bubble, the contact angle was analyzed and calculated using ImageJ software.

Cell culture conditions. Isolation, cultivation and identification of hASCs from the stromal vascular fraction were performed as described in our previous study. The protocols were approved and maintained by the Research Ethics Committee at National Taiwan University Hospital under the guidelines of the Human Subject Research Acts of Taiwan, R.O.C. Informed consent was obtained from human donors. In this study, hASCs (from National Taiwan University Hospital) were cultured in growth medium (DMEM/F-12 (Hyclone, USA) containing 10\% FBS (Biological Industries, USA), 1\% PSA and supplementary 1 ng $\mathrm{mL}^{-1}$ basic fibroblast growth factor (bFGF, Sigma-Aldrich, USA)) in $\mathrm{T} 75$ flask at $37{ }^{\circ} \mathrm{C}$ in an incubator containing $5 \%$ $\mathrm{CO}_{2}$ and saturated humidity. Once the confluence was reached, hASCs were removed from the surface by trypsin-EDTA (Biological Industries, USA), and seeded onto $1 \times 1 \mathrm{~cm}^{2}$ gelatin films in the 24-well plate at the concentration of $10^{4}$ cells per $\mathrm{cm}^{2}$. To avoid cells dropping from the gelatin surface, PDMS pads were put under the gelatin films. After $3 \mathrm{~h}$, PDMS pads were removed and each well was treated with $0.6 \mathrm{~mL}$ medium and the medium was refreshed every 3 days. The cell morphology, viability and proliferation were analyzed.

To induce neural induction, hASCs were cultured in an induction medium (DMEM/HG (Hyclone, USA) containing 1\% FBS and supplementary $100 \mathrm{ng} \mathrm{mL}^{-1} \mathrm{bFGF}$ ) for 7 days. Then cells were cultured in the presence of $10 \mu \mathrm{M}$ forskolin (SigmaAldrich, USA) for another 7 days. The expression of neural differentiation markers was analyzed by immunofluorescence and qPCR.

Cytotoxicity/cell viability. To test the cytotoxicity, the medium in each well was removed and rinsed with PBS two times. After being fixed by $3.7 \%$ formaldehyde solution (ACROS Organics, USA) in PBS for 15 min, cells were stained by using the LIVE/DEAD ${ }^{\text {TM }}$ Viability/Cytotoxicity Kit (molecular probes, USA).
Samples were immersed in solution with $2 \mu \mathrm{M}$ calcein AM and 4 $\mu \mathrm{M}$ Ethidium homodimer-1 (EthD-1) in PBS for 30 minutes.

To test cell proliferation of hASCs on gelatin films, we cultured 5000 cells on nano-grooved and flat gelatin films for 1 , 4 and 7 days. CytoScan ${ }^{\mathrm{TM}}$ WST-1 Cell Cytotoxicity Assay (GBiosciences, USA) was used. With a final volume of $200 \mu \mathrm{L}$ per well culture medium, blank wells with culture medium only were prepared. $20 \mu \mathrm{L}$ WST-1/CEC assay dye solution was added to each well and shook gently to mix the chemicals with the medium. Then, the plates were incubated for $2 \mathrm{~h}$ in the cell culture incubator. After that, the plates were shaken for $1 \mathrm{~min}$ on a shaker and the absorbance at $450 \mathrm{~nm}$ was measured using a microplate reader. The wavelength of $630 \mathrm{~nm}$ was set as a reference.

Cell alignment analysis. At first, $10 \times$ phase contrast microscope images of hASCs cultured on nano-grooved and flat gelatin films were snapped in 20 random areas. To quantify cell alignment, these images were analyzed using ImageJ software. At least 30 cells were analyzed in each image. Cells aligned perfectly in the direction of the grooves have an angle of $0^{\circ}$ and cells perpendicular to the direction of grooves have an angle of $90^{\circ}$. The full range of angles varies between $0^{\circ}$ and $90^{\circ}$. Alignment of cells was determined by measuring the angle between the long axis of cells and the direction of grooves. The experiments were repeated at least three times for each group.

Immunochemistry. After being fixed by $3.7 \%$ formaldehyde solution in PBS for 15 min and permeabilized by 1\% Triton X100 solution (J. T. Baker, USA) in PBS for $10 \mathrm{~min}$ at room temperature, samples were blocked by blocking buffer (5\% BSA solution in PBST, Sigma-Aldrich, USA) at $37{ }^{\circ} \mathrm{C}$ for $1 \mathrm{~h}$. After blocking, samples were immersed in primary antibodies of neural markers including Tuj-1 $\left(2 \mu \mathrm{g} \mathrm{mL}{ }^{-1}\right.$, abcam, USA $)$ and nestin $\left(0.8 \mu \mathrm{g} \mathrm{mL}{ }^{-1}\right.$, abcam, USA $)$ in blocking buffer at $4{ }^{\circ} \mathrm{C}$ overnight. After this, secondary antibodies including Alexa Fluor 488 and 594 (molecular probes, USA) in PBST were used to conjugate with primary antibodies at room temperature for $1 \mathrm{~h}$. Finally, the nucleus was stained with a $10 \mu \mathrm{g} \mathrm{mL}{ }^{-1}$ DAPI solution (Sigma-Aldrich, USA) in DI water for $5 \mathrm{~min}$. Samples were washed extensively by PBST in between each step.

Real-time polymerase chain reaction (qPCR). At first, RNA was isolated by REzol $\left(1 \mathrm{~mL}\right.$ per $10^{6}$ cells, Protech Technology Enterprise, Taiwan), chloroform (Macron Fine Chemicals, USA) and isopropanol (Macron Fine Chemicals, USA). After rinsing with ethanol (Uni-onward, Taiwan), cDNA was produced from RNA via reverse transcription by ARROW-Script RT Mix (Yuanying, Taiwan) and RNase inhibitor (Yuanying, Taiwan).

Then, cDNA, F/R primers (Yuanying, Taiwan), Power SYBR Green PCR Master Mix (Thermo Fisher scientific, USA) and DNase/RNase Free Water (Yuanying, Taiwan) were mixed in Eppendorf PCR tubes (8-tube strips). The sequence of primers is shown in Table 1. After sealing using clear adhesive film, a PCR was run using a real-time PCR instrument (StepOnePlus, Applied Biosystems, USA). The expression level was analyzed and normalized to glyceraldehyde 3-phosphate dehydrogenase (GAPDH) for each sample. The relative quantity (RQ) of gene expression was calculated using the comparative $C_{\mathrm{T}}$ method. The sequence of primers is shown in Table 1. 
Table 1 Primer sequences used in qPCR

\begin{tabular}{lll}
\hline Target gene & & Primer sequences \\
\hline Tuj-1 & Forward & 5'-CATGGATGCCGCTCAG-3' $^{\prime}$ \\
& Reverse & $5^{\prime}$-CAGGCAGTCGCAGTTTCAC-3' \\
Nestin & Forward & $5^{\prime}$-CTCTGACCTGTCAGAAGAAT-3' \\
& Reverse & $5^{\prime}$-GACGATGACACTTACAGAAT-3 \\
GAPDH & Forward & $5^{\prime}$-CAAGGCTGAGAACGGGAAGC-3' \\
& Reverse & $5^{\prime}$-AGGGGGCAGAGATGATGACC-3
\end{tabular}

Statistical analysis. All of the results were expressed as means \pm standard error of the mean. The error bar indicated the standard deviation. Comparison between the different groups was analyzed using Student's $t$-test in Microsoft Excel 2013. This determined statistically significant as a $p$ value $<0.05$ and statistically highly significant as a $p$ value $<0.001$.

\section{Results and discussion}

\section{Topography measurement}

AFM was used to measure nano-grooves on gelatin films (Fig. 2). The distance between adjacent peaks was around $800 \mathrm{~nm}$ on $400 / 100$ and $400 / 400$, and $1800 \mathrm{~nm}$ on $800 / 100$ and $800 / 400$. The vertical distance between the peak and valley was around $150 \mathrm{~nm}$ on $400 / 100$ and $800 / 100$, and 200 to $300 \mathrm{~nm}$ on $400 / 400$ and $800 / 400$. The groove depth of $400 / 400$ was not as deep as $800 / 400$, which may result from the groove with a $400 \mathrm{~nm}$ width being too narrow for gelatin to permeate. Besides, the softness of gelatin and soaring grooves led to a curvature in 2D AFM image of 400/400.

\section{Crosslinking extent measurement}

To determine the crosslinking degree of the gelatin films, TNBS was used to bind with the $\varepsilon$-amino groups in gelatin. It showed yellow and had an absorbance at $346 \mathrm{~nm}$. Less $\varepsilon$-amino groups refers to a higher extent of crosslinking. The result showed that the crosslinking extent of the gelatin films after crosslinking by EDC and NHS for 1 to 4 days rose from $42 \%$ to $57 \%$ (Fig. 3a).

The softness of the gelatin films after crosslinking for 4 days was more suitable for experimental operations. Although the crosslinking extent of gelatin crosslinked by EDC and NHS was not as high as glutaraldehyde ( $\sim 90 \%)$, lower cytotoxicity and proper mechanical strength made it a better choice.

\section{Mechanical test}

The Young's modulus of crosslinked gelatin films was used to determine whether the softness of gelatin is close to natural organs. The Young's modulus of gelatin films after crosslinking for 1 and 4 days was calculated from the linear region of the stress-stain curve (initial slope: $0-10 \%$ ). It was found that the Young's modulus rose from 326 to $894 \mathrm{kPa}$ (Fig. 3b), and was similar to human organs. ${ }^{34}$ Hence, cells were cultured on soft substrates whose stiffness was close to natural organs instead of stiff substrates such as polystyrene and polyurethane.

\section{Swelling test}

Crosslinked gelatin films were bendable and would absorb a lot of water. To investigate when gelatin films stop swelling, the swelling ratio of gelatin films was measured. The results showed that weight of gelatin films crosslinked for 4 days reached $260 \%$ of dry weight after immersing in DI water for $30 \mathrm{~min}$ (Fig. 3c), which means gelatin films had a high water content. On the other hand, it was found that gelatin films can be dehydratedhydrated repeatedly. In this study, all of the gelatin films were immersed in DI water or culture medium for at least $30 \mathrm{~min}$ until use to prevent topography change.



Fig. 2 2D, 3D and cross-sectional AFM images of nano-grooved gelatin films. Scale bar $=2 \mu \mathrm{m} .400 / 100,400 / 400,800 / 100$ and 800/400 (width/depth; $\mathrm{nm}$ ) represent the dimension of four types of grooved substrates. 

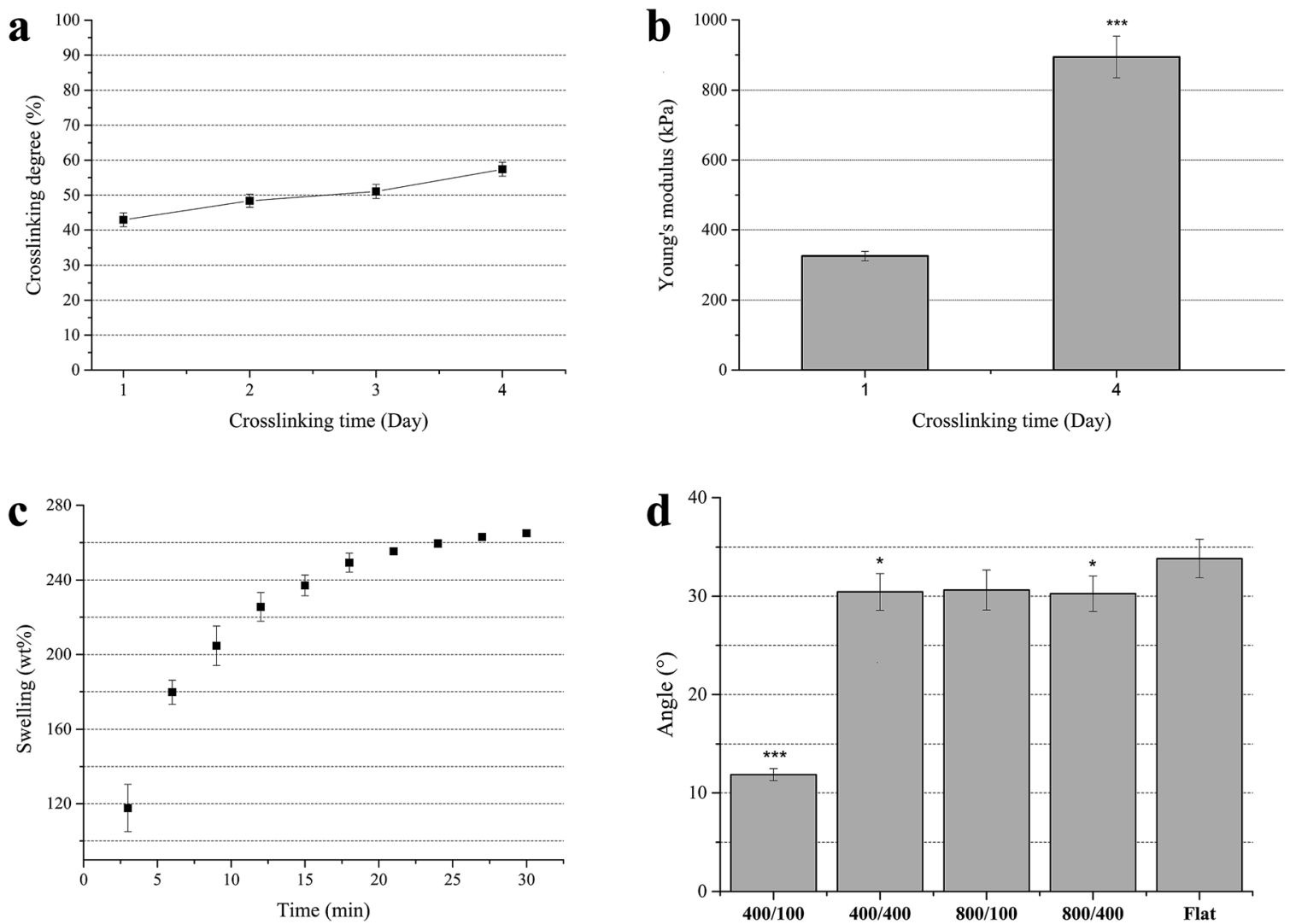

Fig. 3 Features analysis of nano-grooved gelatin films. (a) Crosslinking extent of gelatin films after crosslinking for 1 to 4 days. (b) Young's modulus measurement of flat gelatin films after crosslinking for 1 and 4 days. ***: $p<0.001$, compared to gelatin films after crosslinking for 1 day. (c) Swelling of flat gelatin films after crosslinking for 4 days after different times of storage in DI water. (d) Air bubble contact angle measurement of nano-grooved and flat gelatin films after crosslinking for 4 days. 400/100, 400/400, 800/100 and 800/400 (width/depth; nm) represent the dimension of four types of grooved substrates. *: $p<0.05$ and ***: $p<0.001$, compared to flat gelatin films. All gelatin films were crosslinked by EDC and NHS.

\section{Contact angle measurement}

To investigate the hydrophilicity of nano-grooved gelatin films, the contact angle was measured (Fig. 3d). As gelatin films were immersed in culture medium during cell culture, air bubble contact angle was measured rather than water contact angle. In general, gelatin is hydrophilic and has high air bubble contact angle. The results showed that the contact angle of the air bubble on the 400/100 surface was significantly lower than the other groups, which means the surface of 400/100 was more hydrophobic. A previous study revealed that wettability affects cell adhesion, as cells adhered well on hydrophilic surfaces. $^{35}$

\section{Cytotoxicity/cell viability}

To test the cytotoxicity of gelatin films, the live/dead assay was used for hASCs proliferated on nano-grooved and flat gelatin films for 7 days, and TCPS was used as the control (Fig. 4a). The image showed a massive green fluorescence, which indicates that most of the cells are alive. The ratios of living cells in each group were higher than $90 \%$. This proved that unreacted crosslinking agent had been removed successfully or has only little toxicity to cells.
In addition to cytotoxicity, cell viability was tested using the WST-1 assay (Fig. 4b). hASCs proliferated on nano-grooved and flat gelatin film for 1, 4 and 7 days were tested, and TCPS was used as the control. The results showed cell amounts increase steadily from day 1 to 7 . There is no significant difference between each group except TCPS, which may result from the difference of surface area between the 24 well and gelatin films $\left(1 \times 1 \mathrm{~cm}^{2}\right)$.

\section{Cell alignment analysis}

To observe the cell morphology of hASCs on nano-grooved and flat gelatin films for 1, 4 and 7 days, phase contrast microscope images were taken (Fig. 5a). On day 1, cells were aligned with the grooves on $400 / 400$ and $800 / 400$, whereas part of cells were aligned with grooves on $400 / 100$ and $800 / 100$ until day 7 . It was assumed that groove depth has a greater influence on cell alignment than width.

To quantify cell alignment, the angle between the cell and groove (Fig. 5b) was measured. As mentioned above, about $80 \%$ of cells aligned with grooves within $10^{\circ}$ on $400 / 400$ and $800 / 400$ in the beginning. However, 40 to $55 \%$ of cells on $400 / 100$ and $800 / 100$ seemed to align with grooves within $10^{\circ}$ until day 7 , whereas cells on flat gelatin films still remained in random alignment. 
$\mathbf{a}$
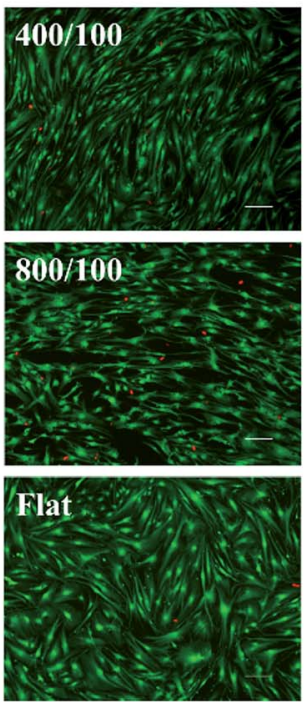

b
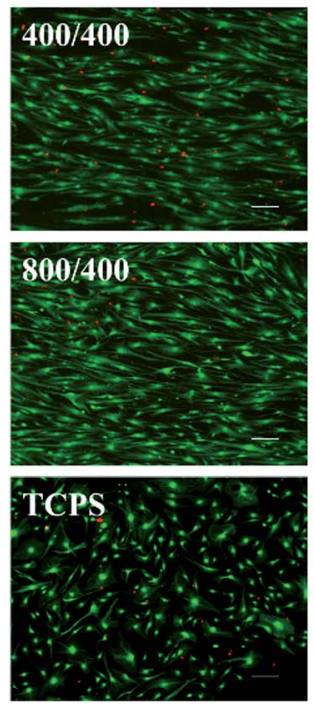

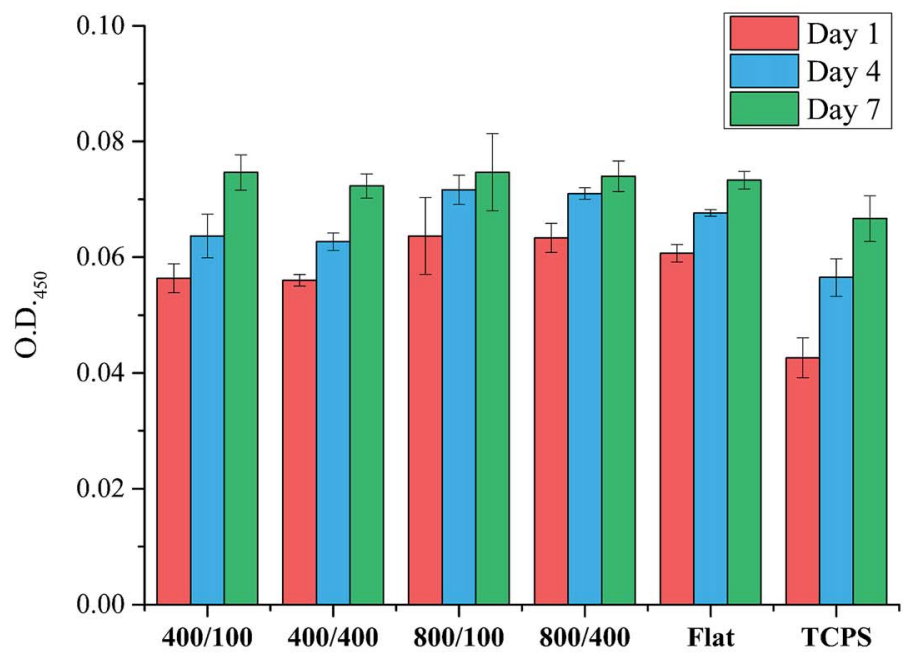

Fig. 4 (a) Live/dead assay of hASCs proliferated on nano-grooved and flat gelatin films after 7 days of culture in growth medium (green: live; red: dead), using TCPS as a control. Scale bar $=200 \mu \mathrm{m}$. (b) WST-1 cell proliferation assay of hASCs proliferated on nano-grooved and flat gelatin films after 1, 4 and 7 days of culture in growth medium, using TCPS as a control. 400/100, 400/400, 800/100 and 800/400 (width/depth; nm) represent the dimension of four types of grooved substrates.

\section{hASCs morphology after neural induction}

Compared to cells proliferated on gelatin films for 14 days, cells differentiated on gelatin films for 14 days showed contraction and neurites outgrowth (Fig. 6a). At the first stage of differentiation, cells were still elongating on day 7 . Then, the cell body gradually contracted and neurites projected. There was no significant difference observed for cell morphology between each group from the phase contrast microscope images.
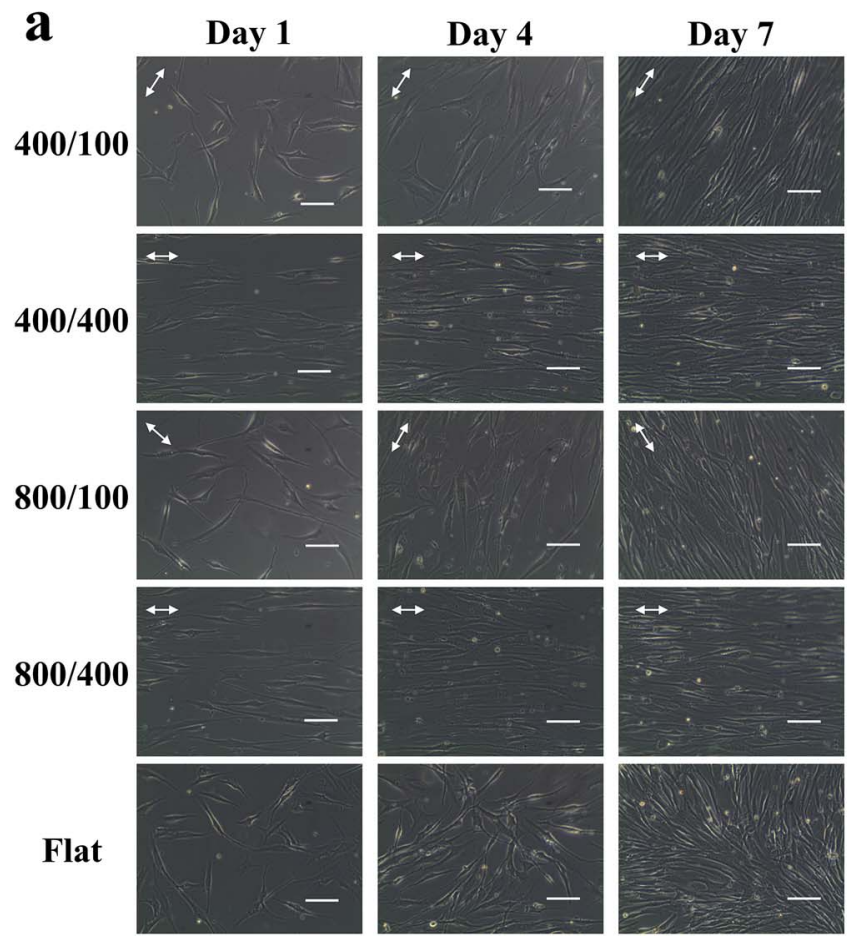

b
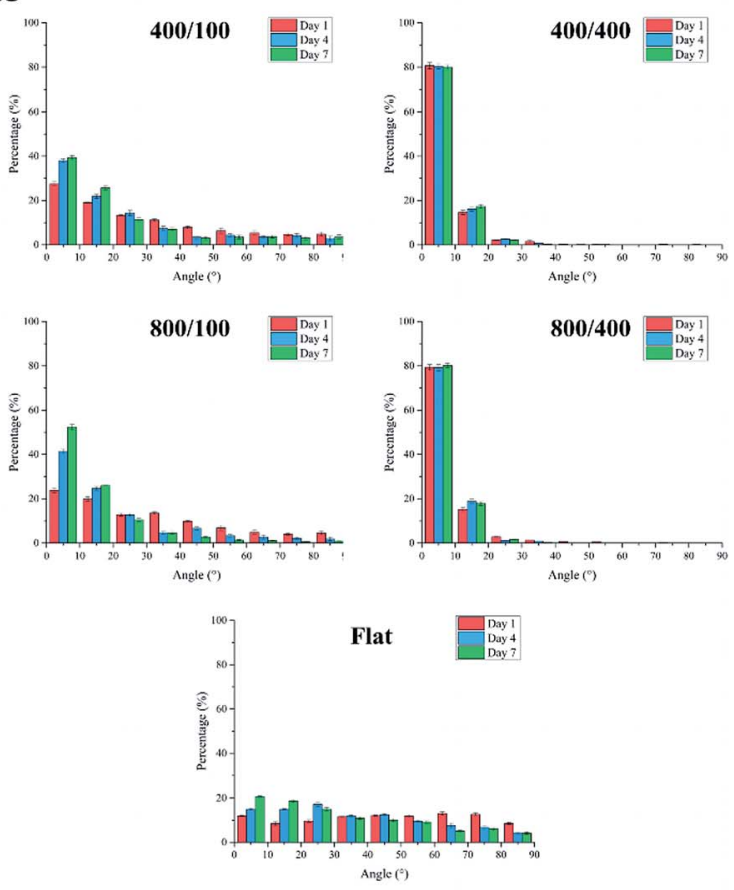

Fig. 5 (a) Phase contrast microscope images and (b) cell alignment analysis of hASCs proliferated on nano-grooved and flat gelatin films after 1, 4 and 7 days of culture in growth medium (scale bar $=100 \mu \mathrm{m}$. The double-headed arrows represent the direction of grooves). (b) Cells aligned perfectly in the direction of grooves have an angle of $0^{\circ}$ and cells perpendicular to the direction of grooves have an angle of $90^{\circ}$. The full range of angles varies between $0^{\circ}$ and $90^{\circ}$. Alignment of cells was determined by measuring the angle between the long axis of the cells and the direction of grooves. 400/100, 400/400, 800/100 and 800/400 (width/depth; nm) represent the dimension of four types of grooved substrates. 
a
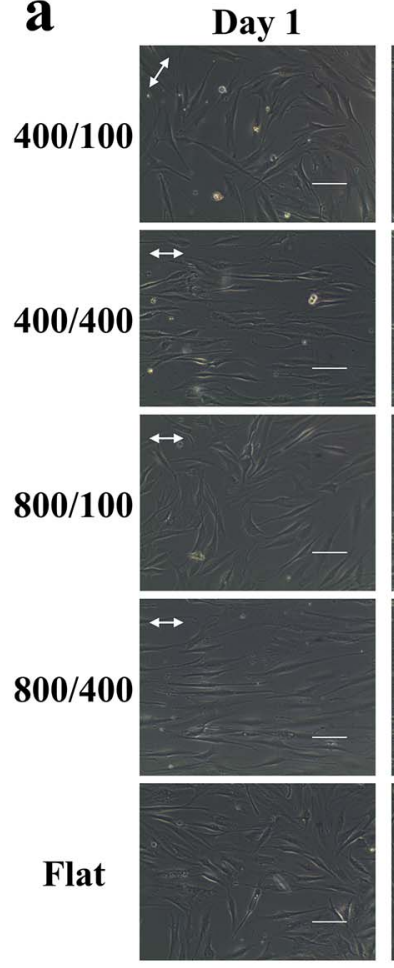

Day 7
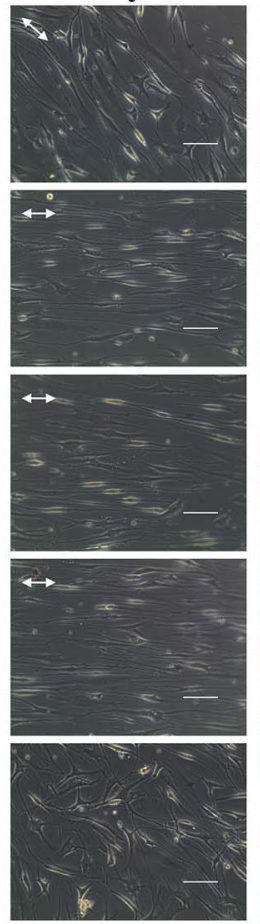

Day 14
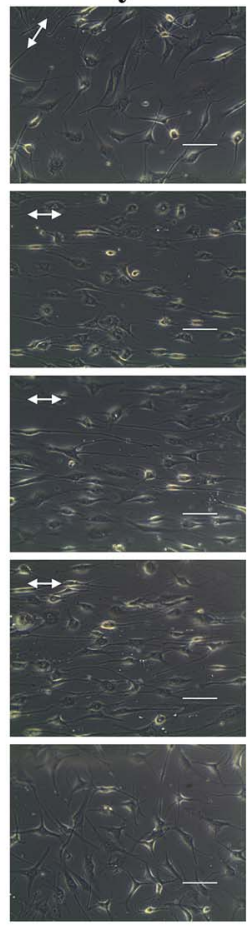

b

b
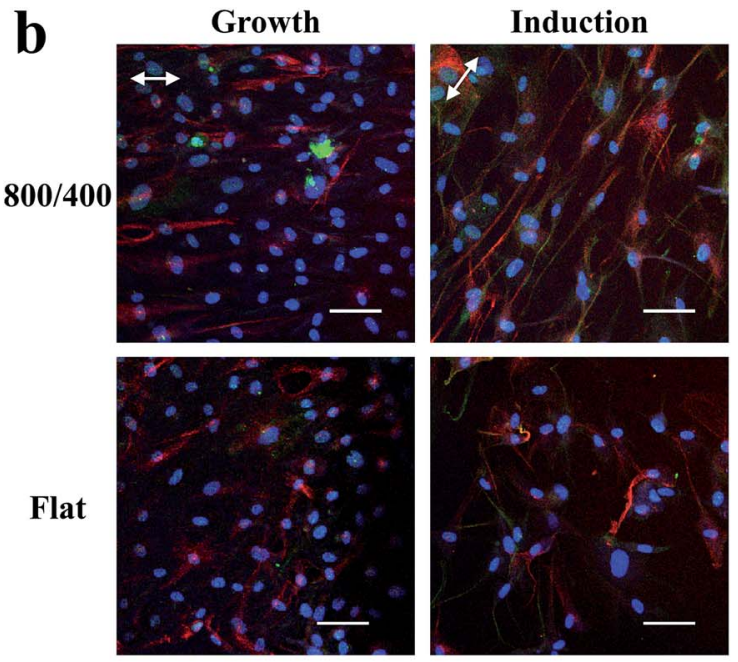

C

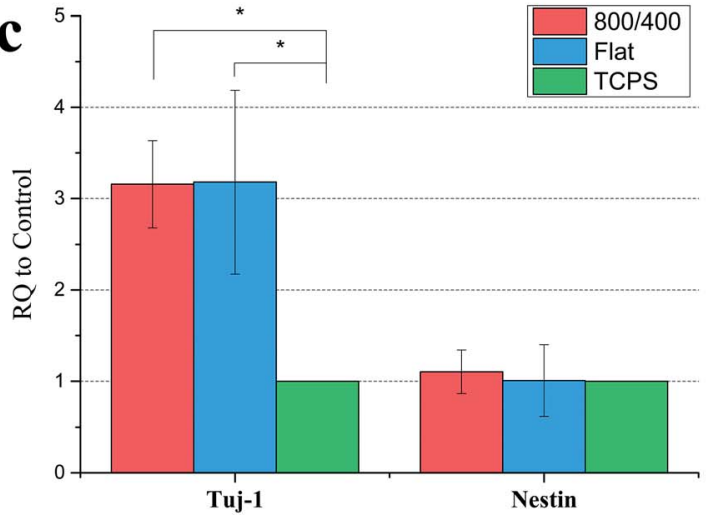

Fig. 6 (a) Phase contrast microscope images of hASCs differentiated on nano-grooved and flat gelatin films for 1, 7 and 14 days in induction medium. Scale bar $=100 \mu \mathrm{m}$. (b) Immunofluorescent staining and (c) qPCR analysis of neural gene expression (Tuj-1 and nestin) of hASCs differentiated on nano-grooved and flat gelatin films after 14 days of culture in induction medium. Cells were stained with DAPI (blue), Tuj-1 (red) and nestin (green). Scale bar $=100 \mu \mathrm{m}$. (c) GAPDH expression of hASCs differentiated on TCPS was used as control. *: $p<0.05 .400 / 100,400 /$ $400,800 / 100$ and $800 / 400$ (width/depth; $n$ m) represent the dimension of four types of grooved substrates. The double-headed arrows represent the direction of grooves.

\section{The expression of neural markers}

To study whether the cells successfully differentiate into neural cells and the effect of grooves on differentiation, we stained Tuj1 and nestin for cells proliferated in growth medium and differentiated in the induction medium on $800 / 400$ and flat gelatin films for 14 days (Fig. 6b). Tuj-1 is a neural marker expressed in immature neurons and nestin is an intermediate filament protein expressed during the early stage of neural differentiation. Confocal images showed a higher ratio of neural marker expression after neural induction, nestin especially. However, there is no difference between $800 / 400$ and flat gelatin films in these images.

To quantify the expression of Tuj- 1 and nestin, we ran qPCR for cells differentiated on 800/400 and flat gelatin films for 14 days with Tuj-1 and nestin (Fig. 6c). The results showed that Tuj1 expression of hASCs on 800/400 was three times higher than TCPS, whereas nestin expression was similar between each group. The increase of the Tuj-1 and nestin expression also correlated with our results from previous studies. ${ }^{36,37} \mathrm{Li}$ et al. demonstrated adult neural stem cells showed a significant increase in neuronal differentiation on engineered anisotropic substrates (Si wafer) compared to the control. In their western blot analysis, upregulated Tuj-1 expression was also seen. ${ }^{36}$ Béduer et al. demonstrated that pre-coated micropatterned PDMS surfaces can serve as effective neurite guidance surfaces for human NSCs. Immunocytochemistry analysis showed that the channel width can strongly impact development and differentiation. ${ }^{37}$

\section{Conclusions}

Topography has shown a great influence on cell behavior in previous studies. Cells were cultured on patterned materials to investigate how topography affects cells. However, most of the materials such as polystyrene (Young's modulus $>3 \mathrm{GPa}$ ) are not as soft as natural tissues. In this study, nano-grooves on gelatin, whose Young's modulus is close to natural organs, was successfully fabricated. Gelatin films are believed to benefit cell adhesion due to their hydrophilic properties and high water content. In addition, gelatin crosslinked by EDC and NHS 
showed low cytotoxicity to hASCs. Cells adhered well on gelatin films and aligned with grooves on 400/400 and 800/400. As for neural differentiation, hASCs contracted and neurites projected after 14 days of induction and immunochemistry and qPCR results revealed that expression of neural markers (Tuj-1 and nestin) was higher after neural induction on gelatin substrates, which are more physical and chemically relevant to real tissue, especially the nano-groove patterned gelatin. Engineered anisotropic topographical cues could improve neurite outgrowth and promote neural differentiation.

\section{Conflicts of interest}

There are no conflicts to declare.

\section{Acknowledgements}

This work was supported by the Ministry of Science and Technology, Taiwan (104-2221-E-002-124-MY3).

\section{Notes and references}

1 B. Saraceno, T. Dua, A. Janca, A. Muscetta, R. Kale, F. Montero and M. Peden, Neurological disorders: public health challenges, WHO, 2006.

2 D. Grinsell and C. P. Keating, BioMed Res. Int., 2014, 2014, 13. 3 M. R. Raffe, Principles of Peripheral Nerve Repair, 1985.

4 S. Walsh and R. Midha, Neurosurg. Focus, 2009, 26, E2.

5 Y. Zhang, A. Gordon, W. Qian and W. Chen, Adv. Healthcare Mater., 2015, 4, 1900-1914.

6 B. N. Mason, J. P. Califano and C. A. Reinhart-King, in Engineering Biomaterials for Regenerative Medicine, ed. S. K. Bhatia, Springer-Verlag, New York, 1st edn, 2012, ch. 2, DOI: $10.1007 / 978-1-4614-1080-5$.

7 D. P. McDaniel, G. A. Shaw, J. T. Elliott, K. Bhadriraju, C. Meuse, K.-H. Chung and A. L. Plant, Biophys. J., 2007, 92, 1759-1769.

8 R. J. Pelham and Y.-L. Wang, Cell Biol., 1997, 94, 1366113665.

9 A. J. Engler, M. A. Griffin, S. Sen, C. G. Bönnemann, H. L. Sweeney and D. E. Discher, Myotubes differentiate optimally on substrates with tissue-like stiffness: pathological implications for soft or stiff microenvironments, J. Cell Biol., 2004, 166(6), 877-887.

10 R. G. Harrison, Science, 1911, 34, 279-281.

11 K. Metavarayuth, P. Sitasuwan, X. Zhao, Y. Lin and Q. Wang, ACS Biomater. Sci. Eng., 2016, 2, 142-151.

12 W. A. Loesberg, J. te Riet, F. C. M. J. M. van Delft, P. Schön, C. G. Figdor, S. Speller, J. J. W. A. van Loon, X. F. Walboomers and J. A. Jansen, Biomaterials, 2007, 28, 3944-3951.

13 J.-Y. Yang, Y.-C. Ting, J.-Y. Lai, H.-L. Liu, H.-W. Fang and W.-B. Tsai, J. Biomed. Mater. Res., Part A, 2009, 90, 629-640.
14 A. G. Sciancalepore, A. Portone, M. Moffa, L. Persano, M. De Luca, A. Paiano, F. Sallustio, F. P. Schena, C. Bucci and D. Pisignano, Biomaterials, 2016, 94, 57-69.

15 M. Kim, A. Habiba, J. M. Doherty, J. C. Mills, R. W. Mercer and J. E. Huettner, Dev. Biol., 2009, 328, 456-471.

$16 \mathrm{~W}$. Wongpaiboonwattana and M. P. Stavridis, J. Visualized Exp., 2015, 52823, DOI: 10.3791/52823.

17 J.-H. Chuang, L.-C. Tung and Y. Lin, World J. Stem Cell., 2015, 7, 437-447.

18 Y.-Q. Liu, L.-B. Zhan, T.-T. Bi, L.-N. Liang, X.-X. Sun and H. Sui, RSC Adv., 2016, 6, 34959-34969.

19 H. W. Choi, Y. J. Hong, J. S. Kim, H. Song, S. G. Cho, H. Bae, C. Kim, S. J. Byun and J. T. Do, PLos One, 2017, 12, e0170735.

20 B.-S. Moon, J.-Y. Yoon, M.-Y. Kim, S.-H. Lee, T. Choi and K.-Y. Choi, Exp. Mol. Med., 2009, 41, 116-125.

21 A. Abdanipour, T. Tiraihi and A. Delshad, Iran. Biomed. J., 2011, 15, 113-121.

22 J. Dong, G. Zhu, T.-c. Wang and F.-s. Shi, J. Zhejiang Univ., Sci., B, 2017, 18, 445-448.

23 H. Ning, G. Lin, T. Fandel, L. Banie, T. F. Lue and C.-S. Lin, Differentiation, 2008, 76, 488-494.

24 P. J. Kingham, D. F. Kalbermatten, D. Mahay, S. J. Armstrong, M. Wiberg and G. Terenghi, Exp. Neurol., 2007, 207, 267-274.

25 J. H. Choi, E. Bellas, G. Vunjak-Novakovic and D. L. Kaplan, Meth. Mol. Biol., 2011, 702, 319-330.

26 Y. S. Choi, G. J. Dusting, S. Stubbs, S. Arunothayaraj, X. L. Han, P. Collas, W. A. Morrison and R. J. Dilley, J. Cell Mol. Med., 2010, 14, 878-889.

27 L. de Girolamo, M. F. Sartori, W. Albisetti and A. T. Brini, J. Tissue Eng. Regener. Med., 2007, 1, 154-157.

28 A. A. Hamid, R. B. H. Idrus, A. B. Saim, S. Sathappan and K.-H. Chua, Clinics, 2012, 67, 99-106.

29 S. Jang, H.-H. Cho, Y.-B. Cho, J.-S. Park and H.-S. Jeong, BMC Cell Biol., 2010, 11, 25.

30 A. Deiwick, E. Fadeeva, L. Koch, R. Gebauer, B. Chichkov and S. Schlie-Wolter, J. Nanomed. Nanotechnol., 2014, 5, 1000239.

31 A. Mobasseri, A. Faroni, B. M. Minogue, S. Downes, G. Terenghi and A. J. Reid, Tissue Eng., Part A, 2015, 21, 1152-1162.

32 J.-Y. Lai, Y.-T. Li, C.-H. Cho and T.-C. Yu, Int. J. Nanomed., 2012, 7, 1101-1114.

33 I. Clyde, M. Ofner and W. A. Bubnis, Pharm. Res., 1996, 13(12), 1821-1827.

34 T. R. Cox and J. T. Erler, Disease Models \& Mechanisms, 2011, 4, 165-178.

35 H. H. Ahn, I. W. Lee, H. B. Lee and M. S. Kim, Int. J. Mol. Sci., 2014, 15, 2075-2086.

36 L. Qi, N. Li, R. Huang, Q. Song, L. Wang, Q. Zhang, R. Su, T. Kong, M. Tang and G. Cheng, PLoS One, 2013, 8, e59022. 37 A. Béduer, C. Vieu, F. Arnauduc, J.-C. Sol, I. Loubinoux and L. Vaysse, Biomaterials, 2012, 33, 504-514. 